INVESTIGACIONES

\title{
FACTORES DE LA ORGANIZACION ESCOLAR QUE CONFIGURAN EL COMPROMISO ORGANIZACIONAL AFECTIVO DE LOS PROFESORES DE ENSEÑANZA BASICA
}

\author{
Factors of the scholar organization that form the affective organizational commitment of \\ preparatory school teachers
}

\section{Guillermo Zamora P.}

Avda. Vicuña Mackenna 4860, Macul, Santiago. gzamora@puc.cl

\section{Resumen}

El presente estudio examina los factores de la organización escolar que se relacionan con el compromiso organizacional afectivo que desarrollan los profesores de enseñanza básica de la ciudad de Santiago de Chile. En términos generales, los resultados indican que los profesores valoran cuatro factores de la organización escolar: la intervención pedagógica con los estudiantes y apoderados, el reconocimiento que reciben a la labor realizada, los recursos educativos que dispone la escuela y las condiciones administrativas. De éstos, el único factor que se relaciona con el compromiso organizacional afectivo es el "recurso educativo" que dispone la escuela. Este factor hace referencia a las condiciones del trabajo pedagógico, las competencias de los colegas y el perfil académico de los alumnos que atiende el establecimiento. El estudio finaliza discutiendo las dificultades de que los alumnos se transformen en una condición laboral de la escuela.

Palabras clave: profesores de enseñanza básica, compromiso organizacional afectivo, organización escolar.

\begin{abstract}
The present study examine the factors of the school organization which are related to the organizacional commitments that are developed by preparatory school teachers of Santiago, Chile. In general terms, the results indicate that teachers valorate four factors of the school organization: the pedagogical intervention with students and parents; the recognition they receive for the work done, the educational resources in the school has, and the administrative conditions. From these, the most relevant factor in the relation with the affective organizacional commitment is "educational resources" that the school has. This factor refers to the conditions of the pedagogic work, the competitions of the colleagues and the academic profile of the pupils of the school. The study finishes discussing the difficulties that imply that the students' become the "educational resource" of the school.
\end{abstract}

Key words: preparatory school teachers, affective organizacional commitment, factors of the school organization. 


\section{INTRODUCCION}

La permanencia de los profesores y profesoras ${ }^{1}$ en los establecimientos escolares es un fenómeno social extraño y peculiar. Desde que se es miembro de un establecimiento, las posibilidades de abandono, renuncia y despido, son infinitas. Es que el permanecer no constituye un estatus socialmente necesario, pese a lo inevitable que se pueda presentar. Más extraña aún es esta permanencia si se consideran los altos índices de malestar docente, lo cual podría ir acompañado de altas tasas de abandono. Hay que considerar que la actividad docente implica un desgaste emocional, físico y mental tan intenso que algunos lo comparan con el del oficio del chofer de locomoción colectiva (Castro 2000). Sin embargo, y pese a todo ello, la permanencia acontece, y en una significativa cantidad de años. De acuerdo a los registros de la encuesta CASEN, los profesores chilenos permanecen 12,8 años bajo el mismo empleador. Esta permanencia, en el caso de los profesores municipales, aumenta a 14,3 años, siendo un 50\% superior que el resto de los profesionales del sector público.

El presente estudio se dirige justamente a comprender la extraña vinculación que se desarrolla entre el profesor y la escuela. Para ello se examina el compromiso organizacional afectivo (COA) que desarrollan los profesores de enseñanza básica hacia sus establecimientos y los factores de la organización escolar que más pueden influir en la conformación de este compromiso.

El compromiso organizacional afectivo (COA) da cuenta del nivel de adhesión e identificación emocional que establece el profesor con su escuela. Este compromiso opera mediante una evaluación directa y simple del grado de satisfacción que experimenta la persona con su organización: cuán contento o no está en ella. Cabe señalar que el COA constituye la dimensión más relevante para explicar la decisión de permanecer de los profesores. Por consiguiente, examinar los factores de la organización escolar que pueden afectar el grado en que se presenta el COA permite identificar los aspectos organizacionales que logran ser altamente valorados por el profesorado y adquieren relevancia para decidir su permanencia: ¿el trabajo pedagógico con los alumnos?, ¿la remuneración que recibe?, ¿el reconocimiento del jefe? Asimismo, indagar acerca de ellos ayuda a comprender cuál es la escuela real que se presenta a los profesores, aquélla con la que se involucra y, por consiguiente, aquélla que le hace sentido para cualquier mejora educativa.

Bajo este marco, el examen de la decisión de permanecer adquiere relevancia puesto que ayuda a comprender una dimensión crucial de la relación de la persona con su organización. Examinar ésta, tal como lo indican Luhmann y De Georgi (1993), implica abordar la primera y fundacional decisión de la persona en su lugar de trabajo. $\mathrm{Su}$ relevancia se sostiene en que esta decisión hace posible y configura -a modo de premisa- otras múltiples decisiones organizacionales, que son vitales para la mejora de la calidad educativa de las escuelas.

De acuerdo a lo anterior, el problema del presente estudio se expresa en la siguiente interrogante:

¿Qué factores de la organización escolar ayudan a configurar el compromiso organizacional afectivo de los profesores?

1 De aquí en adelante se denominará al profesor y/o a la profesora con el genérico "profesor". 
Los objetivos del estudio son los siguientes:

- Identificar y describir los factores de la organización escolar que más valoran los profesores.

- Identificar y analizar los factores de la organización escolar que más se relacionan con el compromiso organizacional afectivo que desarrollan los profesores.

\section{MARCO TEORICO}

1. Decisión de Permanecer y Compromiso Organizacional Afectivo. El estudio de la decisión permanencia en una organización tiene una larga data en las investigaciones laborales. Una de las primeras aproximaciones la realiza Becker (1960), quien considera que en la decisión de permanecer se realizan fundamentalmente cálculos de las pequeñas inversiones -side bets- realizadas por el trabajador a lo largo del tiempo que lleva en la organización. Los "side bets" son las inversiones valoradas por la persona que serían perdidas si éste dejara la organización. Esta perspectiva tiene relación con el compromiso calculativo que plantea Etzioni (1961). Este último postula que en la decisión de permanencia operan cálculos del coste que significaría al trabajador el abandono, producto tanto de lo que deja como de lo que obtendría en una nueva alternativa.

Bajo el anterior esquema, la intención de permanecer aparece como un cálculo de costo y beneficio. Ello implica que el trabajador realiza una decisión eminentemente evaluativa, donde conoce una amplitud de alternativas y tiene claridad acerca de los criterios necesarios para comparar éstas.

Sin embargo, el decidir la permanencia no siempre puede tomar la forma de un cálculo ampliamente informado. March y Simon (1977) observan que este tipo de decisión no sólo requiere de amplia información, sino también de una amplitud de tiempo que haga posible reunir esta información y deliberar en forma detenida cada alternativa. Por ello, agregan, este tipo de decisión es poco frecuente en los ámbitos laborales donde prima la escasez de tiempo y la incertidumbre.

Como una alternativa distinta de las decisiones calculativas, los autores plantean la intención de permanencia como una "decisión satisfactoria". En ésta, a diferencia de la decisión calculativa, no se dispone de una amplia gama de alternativas, ni se hacen exhaustivas evaluaciones entre unas y otras; sino más bien, la persona "se contenta con", sin plantearse lo óptimo o lo máximo, como sucede en la decisión calculativa. Bajo esta perspectiva, la persona decide su intención de permanencia porque le es suficientemente satisfactorio seguir trabajando ahí, sin mayores consideraciones evaluativas del mercado laboral y las posibles consecuencias del cambio. Asimismo, bajo este esquema, la intención de abandono deviene cuando la persona deja de "contentarse con", es decir, cuando la organización ya no le es "suficientemente buena".

De acuerdo a lo anterior, la decisión satisfactoria está menos abierta al mercado y más atenta al vínculo afectivo que establece directamente el trabajador con su organización. En este sentido, lo satisfactorio constituye un examen emocional de lo que le produce la organización.

Consignando la relevancia de la dimensión emocional, es importante destacar los estudios del compromiso organizacional de Meyer y Allen (1984; 1990). Estos autores 
consideran que en la decisión de permanencia se presentan elementos afectivos, normativos y calculativos. Cada uno de ellos configura un tipo de compromiso hacia la organización. El compromiso organizacional afectivo (COA) releva consideraciones de apego emocional hacia el lugar de trabajo; el normativo releva la obligación moral que significa el permanecer; el calculativo releva el coste del abandono y consideraciones evaluativas del mercado laboral.

Existe coincidencia, entre diversas investigaciones, en considerar que el COA es de los tres compromisos el más determinante para decidir permanecer (Meyer, Allen, y Smith 1993; Jaros 1997; Zamora 2005). Es interesante destacar el estudio de Zamora (2005) a profesoras de enseñanza básica de la ciudad de Santiago de Chile. Esta investigación consigna una correlación entre intención de permanecer y COA de $\mathrm{r}=0.582$ (sig. menor de 0,05). Este valor supera cuatro veces el poder explicativo entre compromiso calculativo e intención de permanencia.

A partir de lo anterior, es posible señalar que la decisión de permanecer está estructurada primordialmente por una vinculación emocional que establece el profesor hacia su escuela. La dimensión afectiva involucrada hace referencia a la adhesión sentimental y el grado de gusto -o disgusto- que le provoca la organización. Ello significa que los profesores tienden a decidir su permanencia como una evaluación directa y simple de su estado emocional con la escuela, y escasamente se utilizan estrategias comparativas de las ofertas del mercado laboral.

\section{Factores de la organización escolar asociados al compromiso organizacional afectivo.} Los estudios que han examinado los factores y dimensiones del lugar de trabajo que se asocian al COA provienen fundamentalmente del medio anglosajón. Ellos tienden a coincidir que el desarrollo del trabajo mismo, la ejecución de éste, constituye el factor más determinante para la configuración del COA.

Al respecto, Kushman (1992) en un estudio a profesores de escuelas rurales y urbanas de Norteamérica, concluye que el COA es muy sensible a la obtención de experiencias de logros de aprendizajes con los alumnos. Según el investigador, el COA tiende a hacerse más pronunciado cuando los profesores perciben que tienen control sobre los procesos de enseñanza, cuando experimentan eficacia sobre su trabajo y cuando tienen más experiencias de éxito instruccional. En otras palabras, el profesor se siente entusiasmado cuando los alumnos se entusiasman y avanzan en sus aprendizajes. Esta situación les permite confirmar su efectividad y su valor profesional.

El estudio de Rosenholtz y Simpson (1990), a 1.213 profesores primarios de Estados Unidos, también confirma la relevancia del trabajo académico en el desarrollo del COA. Los investigadores observan que la conformación del COA está influida primordialmente por la disciplina escolar en el aula y el manejo de la autoridad pedagógica de los profesores. De este modo, los autores concluyen que el principal antecedente del COA se puede resumir en una gran variable: el grado de satisfacción por cumplir profesionalmente con la tarea del trabajo pedagógico.

En esta misma línea, Rosenblatt (2001), en un estudio a profesores de escuelas primarias de Israel, consigna que la variedad y satisfacción del trabajo pedagógico es uno de los antecedentes más significativos para la configuración del COA. Los profesores más comprometidos perciben más variedad en sus trabajos, lo cual les permite ejercitar sus destrezas, obteniendo así mayores experiencias de logro. 
En el medio latinoamericano, las investigaciones al respecto presentan un cuadro más complejo. Si bien hay estudios que destacan la labor pedagógica como uno de los factores más valorados por los docentes, también hay otros que indican que ellos centran más su atención en las relaciones interpersonales y las condiciones laborales.

Con respecto a la diversidad de los factores más valorados, Gatti, Espósito y Da Silva (1994), en Brasil, y Mendizábal (1995), en Argentina, coinciden en que éstos son tanto las actividades de la sala de clases, como también las buenas relaciones con los colegas y directivos.

Corroborando la pluralidad de los factores, es importante considerar el estudio de salud mental de los profesores chilenos de Méndez y Bernales (1996). Los investigadores examinan las percepciones de los docentes acerca de las principales causas de estrés. Este diagnóstico da luces sobre los aspectos laborales más relevantes para los docentes. Los resultados indican que los profesores que se inician en la profesión destacan, en primer lugar, la indisciplina de los alumnos y la falta de control sobre ellos. En segundo lugar, las condiciones laborales: horarios y sueldo; y en tercer lugar, los aspectos sociopolíticos del contexto: cambios en los programas ministeriales, nuevos requerimientos sociales.

También es interesante destacar el estudio de Cornejo y Rodríguez (1995). En esta investigación se examinan a profesores del sector municipalizado. Se utiliza un cuestionario cerrado que solicita que los profesores indiquen el grado de importancia que tienen para su satisfacción general tres grupos de factores organizacionales. El grupo de factores más relevante se refiere a la práctica docente $(96 \%$ señala que es importante para su satisfacción); en este grupo destaca la valoración del trabajo realizado por los alumnos, el desarrollo de destrezas intelectuales y la posibilidad de desarrollar relaciones cálidas con los estudiantes. Esta situación es explicada por los autores en cuanto los maestros hacen referencia a aspectos en los cuales tienen mayor control y dependen de ellos. El segundo grupo de factores se refiere a los roles y responsabilidades laborales ejercidas ( $82 \%$ los considera importantes para su satisfacción). Por último, se presenta el grupo de factores relacionados con las condiciones laborales (72\% la destacan).

De los anteriores antecedentes se puede conjeturar que los profesores latinoamericanos mantienen una percepción amplia y diversa de los factores organizacionales que son más valorados. Ciertamente la labor pedagógica es muy relevante, sin embargo no es la única, y quizás tampoco la principal. Las condiciones de trabajo y las relaciones interpersonales con los pares adquieren una significativa relevancia.

Por último, es necesario comentar que hasta la fecha no se han encontrado en Chile estudios que examinen directamente la relación entre factores organizacionales y el COA. Dada esta complejidad, es que se hace aún más relevante el examinar la realidad educacional chilena, identificando claramente los factores organizacionales que ayudan a configurar el compromiso organizacional afectivo.

\section{METODOLOGIA}

1. Diseño de la investigación. El diseño de esta investigación es de tipo descriptivo y correlacional, su pretensión es dar cuenta del comportamiento y relaciones entre las variables medidas, mediante técnicas estadísticas y de análisis de contenido. 
2. Muestra. Este estudio fue realizado con los datos obtenidos de 513 profesores de enseñanza básica, de la ciudad de Santiago, pertenecientes a establecimientos de dependencia municipal, particular subvencionada y particular pagada. Los sujetos encuestados son profesores jefes de $1^{\circ}$ a $8^{\circ}$ E.B. Se han excluido de la muestra aquellos docentes que asumen cargo directivo o administrativo

La muestra es aleatoria estratificada, de afijación uniforme por cada dependencia escolar. Ello se debe a la necesidad de asegurar una adecuada diversidad de realidades organizacionales.

Las características sociodemográficas de la muestra coinciden con las características poblacionales. El 87,2\% de la muestra son mujeres; la edad promedio es de 44,11 años; tienen en promedio 20,1 años de servicio; llevan trabajando en sus actuales establecimientos 11,77 años promedio.

3. Instrumentos y Variables. Se aplica un cuestionario de percepciones de la organización escolar. La aplicación del instrumento se realiza durante los meses de mayo y junio del año 2005. Se encuesta en forma simultánea, por establecimiento, a todos los profesores jefes de enseñanza básica. La simultaneidad se realiza para evitar efectos de contaminación en las respuestas. El cuestionario es anónimo y voluntario.

A continuación se caracterizan las variables examinadas.

a) Variable Dependiente: Compromiso Organizacional Afectivo.

Esta variable se examina mediante una escala compuesta por ocho ítemes (tipo lickert) que ha sido confeccionada a partir de la versión anglosajona realizada de Allen y Meyer (1990). Los ítemes son los siguientes:

\begin{tabular}{|l|l|}
\hline 1 & Estaría feliz en continuar el resto de mi vida laboral trabajando en este Establecimiento. \\
\hline 2 & Siento de verdad que cualquier problema de este Establecimiento, es también mi problema. \\
\hline 3 & Siento que tengo un fuerte sentido de identificación hacia este Establecimiento. \\
\hline 4 & Me siento de verdad emocionalmente unido(a) a este Establecimiento. \\
\hline 5 & En este Establecimiento me siento como en familia. \\
\hline 6 & Realmente estoy contento de trabajar en este Establecimiento. \\
\hline 7 & Siento que le debo mucho a este Establecimiento. \\
\hline 8 & Realmente este Establecimiento se merece mi lealtad \\
\hline
\end{tabular}

La escala ha sido previamente validada mediante juicios de expertos. También se ha medido su confiabilidad, la cual reporta un Alpha de Cronbach estandarizado de 0,8588, lo cual es altamente significativo.

b) Variable Independiente: Factores de la organización escolar valorados por los profesores.

Esta variable corresponde a aquellos aspectos y características del establecimiento escolar que son experimentados por el profesor de modo positivo o negativo.

El factor organizacional -positivo o negativo- se recoge desde un incidente crítico que experimenta el profesor y que selecciona como uno de los eventos más relevantes 
que le ha ocurrido en el último año en su establecimiento. Con este procedimiento se asegura que el factor identificado sea genuinamente relevante para los propios profesores. Para levantar el incidente crítico se plantean, en el cuestionario, dos preguntas abiertas. Ellas han sido formuladas teniendo en cuenta el estudio de Herzberg (1966). Las preguntas fueron validadas por los 7 jueces externos.

Las preguntas formuladas son las siguientes:

"Recuerde Ud. una ocasión que haya tenido lugar durante el último año, en que se haya sentido excepcionalmente mal o en que se haya sentido excepcionalmente bien en su trabajo. Elija sólo una situación.”

a. Por favor, describa detalladamente aquella situación.

b. ¿Qué le significó a Ud. esta situación? Por favor responda lo más a fondo posible.

La identificación de los factores laborales más relevantes para los docentes se realiza mediante la técnica de análisis de contenido centrado en el nivel semántico.

4. Técnicas de análisis de resultados. Para examinar la relación entre factores de la organización escolar valorados por los profesores y el compromiso organizacional afectivo, se realizan dos análisis: correlación de Tau-b de Kendall y regresión logística.

La correlación de Tau-b de Kendall es un modelo que aporta un coeficiente de asociación para variables de rangos que tiene en consideración los empates. Al igual que el coeficiente $\mathrm{r}$ de Pearson, indica la dirección de la relación; y su valor indica la magnitud de la misma. Los valores pueden oscilar entre -1 y 1 .

Para este análisis se ha ingresado un valor para todos los sujetos de la muestra, considerando tanto la orientación (positiva y negativa) del factor, como también la mención de él. Específicamente se establecen para cada sujeto las siguientes puntuaciones en cada uno de los factores incluidos:

\footnotetext{
$-1=$ El factor es señalado con orientación negativa

$0=\mathrm{El}$ factor no es señalado

$1=\mathrm{El}$ factor es señalado con orientación positiva.
}

Esta puntuación permite establecer un valor para cada sujeto en los respectivos factores, logrando así la inclusión de todos los sujetos de la muestra.

Posteriormente se aplica un análisis de regresión logística, el cual permitirá examinar si los factores laborales, de acuerdo a su orientación positiva o negativa, establecen alguna diferencia en relación al grado en que se experimenta el COA. La elección de este tipo de análisis se sustenta en la distribución asimétrica -concentrados en valores positivos- de la variable COA. La aplicación de otro tipo de regresión -por ejemplo el lineal- se dificulta por el incumplimiento del requisito de homocedasticidad y normalidad de los residuos.

Cabe señalar que la regresión logística es una técnica que tiene el propósito de pronosticar la pertenencia a una categoría de la variable dependiente a partir del comportamiento en la variable independiente. En este caso, permite identificar el poder de 
cada factor -en su orientación positiva o negativa- para predecir el que se tenga un alto o bajo COA. Una de las cualidades de la regresión logística es que permite utilizar variables dependientes e independientes categóricas. Esto soluciona la problemática de distribuciones asimétricas. Por último, hay que señalar que se han respetado los supuestos estadísticos de la aplicación de esta técnica. Particularmente, el tamaño de la muestra y el evitar efecto de colinealidad.

\section{RESULTADOS}

Siguiendo los objetivos planteados en esta investigación, a continuación se presentan los resultados para cada uno de ellos.

1. Factores de la organización escolar más valorados. En términos generales, se recogen 497 relatos -incidentes críticos-, que representan el 96,8\% de los profesores de la muestra. Sólo 16 sujetos no responden las preguntas planteadas.

Es igualmente importante mencionar la equilibrada proporción de relatos de orientación positiva (262, lo que representa el $52,7 \%$ de la muestra) y negativa (235, que representa el $47,3 \%$ de la muestra).

Aplicado el análisis de contenido, se logran distinguir cuatro grandes factores organizacionales que valoran los docentes: la intervención pedagógica con los estudiantes y apoderados (logro pedagógico), el reconocimiento a la labor realizada, los recursos educativos y las condiciones administrativas. Acerca de cada uno de ellos, se comenta a continuación.

a) Intervención o Logro Pedagógico. El factor "logro Pedagógico" reúne a todos los relatos que hacen alusión a la consecución de un desempeño en los alumnos o en los apoderados a partir de la intervención del propio profesor (el que narra el incidente crítico).

En términos de frecuencia, este factor representa el 34,1\% de todos los relatos. En su mayor proporción, se presentan con una orientación positiva ( $26 \%$ de todos los relatos). Los de orientación negativa presentan una frecuencia de sólo un 8,1\%.

En estos relatos, el actuar del profesor adquiere un rol muy activo, puesto que él asume que los desempeños que logra en los alumnos y en los apoderados es una consecuencia de su propia acción. En tal sentido, los desempeños obtenidos (o no obtenidos, en caso negativo) constituyen una experiencia de logro o no logro personal.

Un relato que presenta claramente el logro pedagógico es el siguiente (relato $\mathrm{N}^{\circ} 1$ ). Aquí una profesora da cuenta de un apoyo didáctico y la consecuente mejoría en el alumno. La profesora declara que esta situación le genera una sensación de eficacia por haber "logrado sacar fuera su potencial [del alumno]". La experiencia se refiere a la efectividad de su consecución. Ello se observa claramente en el significado que le atribuye: autoafirmación de que lo que hago tiene frutos. 


\section{Relato $\mathrm{N}^{\mathrm{o}} 1$}

[Situación] "Año pasado, cuando un alumno que venía en mi asignatura con un promedio inferior a 3 , realicé un apoyo en la clase, fuera de ella, apoyándolo básicamente sus potencialidades. Resultado obtuvo nota 6,2 en una evaluación. Me sentí muy, pero muy contenta de haber logrado sacar fuera su potencial. [Significado] Una autoafirmación de lo que hago tiene sus frutos, que la línea que pretendo seguir tiene resultados a corto plazo y visibles para los niños. Me motivó a seguir estudiando, conociendo formas de poder ayudar a los niños." (Profesora $7^{\circ}$ E.B., 40 años. Establecimiento Particular Pagado, Simce Alto).

En los relatos de orientación negativa, los significados que le atribuyen los profesores tienen el mismo patrón de los positivos pero en sentido inverso. Los profesores señalan que cuando los alumnos (o sus apoderados) no presentan los desempeños esperados, ellos se sienten "fracasados", "disminuidos en el valor de su eficacia", "impotentes", "poco significativos". De este modo, el problema de sus alumnos (o de sus apoderados), se transforma en un problema de eficacia personal, presentándose como una experiencia de no logro: el profesor intenta, pero no puede.

Este aspecto se observa claramente en el siguiente relato $\left(\mathrm{N}^{\circ} 2\right)$. Aquí la profesora describe un desempeño escolar inadecuado que no logra revertir. Dado que está en juego su intervención, esta situación le genera un profundo sentimiento de frustración por su ineficacia. La estructura del relato se puede sintetizar como: la profesora interviene, pero no hay modificación del entorno. Frente a ello aparece la experiencia frustración, que ella la denomina como "impotencia".

\section{Relato $\mathrm{N}^{\mathrm{o}} 2$}

[Situación] "Un día en clases de lenguaje, dada la actitud de los alumnos de $7^{\circ}$ año, era casi imposible llevar el desarrollo de la clase como estaba planificada. Los niños estaban totalmente alterados, agresivos, muy bulliciosos; era como tratar de hablar a un grupo de personas que estaban en una revuelta. Era impresionante observar la anarquía que se daba en la sala. [Significado] Me sentí frustrada, impotente, no encontraba las herramientas que me ayudaran a revertir esa situación. Sentí deseos de salir arrancando, de irme lo más lejos posible y de no volver. Es muy frustrante no poder realizar una actividad que está planificada, organizada, con el material necesario y observar que los alumnos no tienen hábitos ni respeto, y que no tienen claro lo importante que es para ellos la educación formal, sobre todo en un sector tan deprivado." (Profesora, 35 años, $7^{\circ}$ E.B., Establecimiento Municipal, Simce Bajo).

b) Reconocimiento a la labor realizada. El factor "reconocimiento" reúne a los relatos que hacen alusión a situaciones en que un miembro del establecimiento (alumno, apoderado, colega o directivo) emite algún tipo de evaluación al profesor. Éstas pueden ser expresiones manifiestas, como un elogio o crítica, o expresiones más implícitas, como un obsequio o una indiferencia. Las evaluaciones se presentan a partir de una conducta específica del profesor o por una cualidad general que ha presentado durante un tiempo más prolongado. 
Este factor presenta el 36,8\% de todos los relatos clasificados. Existe una proporción similar entre relatos de orientación positiva y negativa (18,3 y 18,5\% respectivamente).

Los profesores interpretan el reconocimiento como una expresión de valoración personal. En el caso de los relatos de orientación positiva, los maestros suelen señalar que tales manifestaciones les hacen "sentirse queridos", "valorados como seres humano", "importantes como persona". En todos estos casos, los profesores perciben la evaluación como una retroalimentación o recompensa de la acción que han ejercido. Esta interpretación se aprecia claramente en el siguiente relato $\left(\mathrm{N}^{\circ} 3\right)$.

\section{Relato $N^{\circ} 3$}

[Situación] "Excepcionalmente bien cuando me correspondió organizar una vigilia con los alumnos con motivo de celebración del día de nuestra Congregación y por primera vez mi directora me felicitó. [Significado] Me significó una gran motivación a continuar realizando otras actividades y además sentirme una "persona" valiosa, no tan sólo por la parte académica y profesional. Esto no significa buscar reconocimiento público ni económico, sino más bien que con el abrazo que ella me dio me sentí con la autoestima muy alta." (Profesora, 42 años, $6^{\circ}$ E.B. Establecimiento Particular Pagado, Simce Bajo).

Aquí la profesora atiende a las felicitaciones recibidas, especialmente el "abrazo de su directora", como una experiencia única ("por primera vez") de reconocimiento. Lo interesante es que este reconocimiento lo interpreta en términos de valía personal, más que una retroalimentación técnica. De ahí que el abrazo se convierta en una fuente de autoestima personal.

Es interesante observar en este grupo de relatos que la valoración presentada no se interpreta como una expresión gratuita hacia el profesor. Por el contrario, ella adquiere su fuerza gracias a que previamente se han atendido las cualidades o el obrar del profesor. Es decir, hay una valoración que se fundamenta en una distinción o atención que hace la organización al profesor. Por consiguiente, se puede señalar que en estos relatos se presenta un doble mensaje: una distinción y una valoración.

En el caso de los relatos negativos, se presenta una estructura similar. Los profesores perciben que su esfuerzo o sus cualidades no han sido consideradas, o en caso que se les descalifique, ellos consideran que hay un error o injusticia en los aspectos que fueron atendidos. En uno u otro caso, la queja es tanto la desvaloración, como también la equivocada o nula atención que les proporciona la organización a lo que ellos realizan.

c) Recurso Educativo. El factor "Recurso Educativo" reúne a los relatos que hacen referencia a los componentes humanos y organizativos que ofrece y administra la escuela, y que impactan en el contenido del trabajo pedagógico que realiza el profesor. En este factor se hace referencia a la organización del trabajo pedagógico, las competencias de los colegas y el perfil académico de los alumnos que atiende el establecimiento.

Este factor presenta el 17,6\% de todos los relatos clasificados. Existe una proporción relativamente similar entre relatos de orientación positiva $(7,6 \%)$ y negativa $(10 \%)$. 
En cuanto a la organización del trabajo, los profesores hacen referencia a las facilidades y obstáculos que les presenta la organización escolar para realizar sus funciones técnicas y pedagógicas. Incluyen situaciones referidas a la cantidad y tipo de tareas que la gestión le demanda, asistencia técnica, apoyo y directrices que le presenta la dirección y recursos materiales para la enseñanza. Estos dominios son considerados por los profesores como condiciones laborales facilitadoras u obstaculizadoras para realizar su trabajo educativo.

En cuanto a las competencias de los colegas, los profesores hacen referencia a las cualidades personales y técnicas de sus compañeros de trabajo. Los profesores observan el perfil de sus colegas como una buena o mala condición que dispone la organización y que influye en la función pedagógica general de la escuela. En tal sentido, las competencias de sus colegas constituyen una condición estructural que aporta alta o baja calidad educativa.

En cuanto al perfil de los alumnos, los profesores hacen referencia a las competencias académicas que los estudiantes poseen como una facilidad o como un obstáculo para realizar el trabajo pedagógico. El perfil académico es interpretado como una condición -negativa o positiva- que dispone la escuela.

En el caso de los relatos de orientación positiva, los profesores señalan que las favorables cualidades académicas de sus alumnos les producen gran alegría y satisfacción dado que les proporcionan tranquilidad laboral. Un claro ejemplo al respecto es el siguiente relato $\left(\mathrm{N}^{\circ} 4\right)$. Aquí la profesora indica que, producto de las cualidades que actualmente presentan sus alumnos, trabajar con su curso le genera más energía y entusiasmo para el trabajo futuro. Estas cualidades se pueden traducir como una adecuada condición laboral que le permite estar más relajada.

\section{Relato $\mathrm{N}^{\mathrm{o}} 4$}

[Situación] "Actualmente soy profesora jefe en $3^{\circ}$ básico con un grupo de alumnos asertivos, con una buena formación de hábitos y con un ritmo de aprendizaje diferente al $3^{\circ} 2002$. Este curso realmente me ha devuelto la vocación y entusiasmo en mi carrera, por lo cual obviamente trabajo este año contenta, relajada y con mucha energía. [Significado] La situación de este año me parece que se realizó con justicia en relación a la del año pasado y se complementa con la receptividad profesor-alumno-padres. Estoy muy satisfecha." (Profesora, 45 años, $3^{\circ}$ E.B. Establecimiento Particular Pagado, Simce Alto).

En cuanto a los relatos de orientación negativa, ellos se refieren a situaciones en que el profesor observa dificultades en los comportamientos y aprendizajes de su curso. Al respecto, los profesores suelen señalar sentimientos de malestar por la injusticia que significa el atender a estos "malos alumnos". También se indican sentimientos de estrés y tensión dado que se considera que esta situación laboral les demandará un mayor esfuerzo.

Un claro ejemplo al respecto se presenta en el siguiente relato $\left(\mathrm{N}^{\circ} 5\right)$. Aquí la profesora indica el malestar que le significa trabajar con un curso con el que había tenido una mala experiencia. Es interesante que ponga énfasis en la incorporación de un alumno repitente, lo cual le genera agotamiento. En este sentido, el tipo de alumno con que trabaja constituye la expresión de una desfavorable condición laboral. 


\section{Relato $\mathrm{N}^{\mathrm{o}} 5$}

[Situación] "La situación corresponde a este año. La noticia me la dieron en diciembre de 2002 cuando me dijeron que para el 2003 me tocaba un $4^{\circ}$ básico, a lo cual se agregó que antes del inicio de la jornada escolar me enteré que además tenía un alumno repitente. [Significado] Estuve bastante tiempo triste y con rabia porque yo no quería tener a este $4^{\circ}$. No me gustaba el curso en sí, ya que los había tenido hace unos años atrás. El trabajo es agotador. Por otro lado, el alumno repitente era un gran desafío para mí y no me sentía preparada." (Profesora, 26 años, $4^{\circ}$ E.B. Establecimiento Particular Pagado, Simce Medio).

En suma, los tres tipos de relatos mencionados hacen referencia, en forma explícita o implícita, a las condiciones que dispone y administra la organización, y que impactan en el contenido pedagógico del trabajo del profesor. Dado el carácter de condición laboral, es común que en estos relatos el profesor aparezca con un rol más pasivo y observante: ellos se circunscriben a juzgar acerca de la calidad (adecuada o inadecuada, favorable o adversa) de estas condiciones.

También es relevante que los profesores interpreten en estas condiciones las decisiones directivas que las sostienen. Ello se evidencia especialmente en el factor organización del trabajo y competencias del alumno. En estos casos, los profesores suelen hacer juicios acerca de lo justo o injusto de tal condición. Por ejemplo, el nuevo formato de planificación, la distribución de los cursos, el tipo de selección de alumnos. Estas apreciaciones significan que los profesores no sólo están atentos a lo favorable o adverso de las condiciones para realizar el trabajo, sino que también consideran que en estas condiciones están presentes las decisiones -buenas o malas- de la gestión. A ello hay que agregar las interpretaciones acerca de la valoración que ellos creen que está comprometida: "no se ha considerado mi persona", "es una falta de respeto", etc. Estas menciones indican que las condiciones son abordadas en una doble dimensión. Esto es: la estructura que influye en la forma como se hace el trabajo, y la expresión de valor que aparece en la decisión que sostiene a esta estructura.

Ahora bien, es interesante observar que la valoración que se desprende de la administración de las condiciones laborales es distinta a la expresión de reconocimiento. Esta última requería previamente la distinción del hacer del profesor, por lo cual se presentaba como una retroalimentación que era "ganada" por el esfuerzo del profesor. En cambio, en el presente factor, las valoraciones tienen relación más bien con el nivel de estima profesional que le aporta la dirección. En otras palabras, no se trata de una valoración que espera ser ganada, sino que se espera que se garantice en forma incondicional.

d) Condiciones Administrativas. El factor "Condiciones Administrativas" reúne a los relatos que hacen referencia a las condiciones del trabajo referidas a compensaciones económicas y estabilidad laboral.

Este factor representa el $11,6 \%$ de todos los relatos clasificados $(\mathrm{N}=458)$. En mayor proporción se presentan relatos de orientación negativa $(9,8 \%)$. Escasamente se presentan relatos de orientación positiva (1,5\%).

El factor "condiciones administrativas" recompensa la mantención de los profesores en el establecimiento. En tal sentido son más generales que el factor referido a "recurso educativo". A diferencia de éste, que lograban estructurar el contenido del trabajo a 
realizar, el presente factor sólo alude a condiciones de la permanencia. Ciertamente, por la generalidad de estas condiciones, no se excluye la posibilidad que tengan efectos en la estructura misma del trabajo que se realiza.

Estas condiciones administrativas tienen, al igual que el "recurso educativo", la cualidad de ser administrables por las direcciones escolares. Las compensaciones económicas son abordadas como recompensas discrecionales de los propios establecimientos.

La discrecionalidad de las compensaciones económicas también se observa cuando los profesores atribuyen el descenso de sus sueldos como un problema de retribución de sus empleadores a los esfuerzos realizados. De este modo, en este factor también aparece el tema de la valoración profesional. Los profesores tienden a desentrañar en las variaciones salariales los niveles de aprecio que les presenta la dirección.

La estabilidad de la fuente laboral, aunque en un grado menor, también aparece ligada a la discrecionalidad de direcciones escolares. Son ellas las que confirman la permanencia o generan incertidumbre. También en este factor aparecen juicios de los profesores acerca de sus direcciones: tanto por la idoneidad administrativa, como por los niveles de aprecio que hacen de su labor.

Se ha puesto énfasis en esta discrecionalidad puesto que ayuda a comprender que las condiciones administrativas, que relevan los profesores, no sólo dan cuenta de una estructura laboral favorable o adversa para la permanencia en la organización, sino también dan cuenta de una expresión de valoración (aprecio, cuidado) que le comunica la organización.

2. Relación entre factores organizacionales y compromiso organizacional afectivo. Aplicada la correlación de Tau-b de Kendall (tabla 1), se puede observar que los factores que mejor se relacionan con el COA son el "Reconocimiento" $(0,127$, que es significativo al nivel de 0,01$)$ y los "Recursos Educativos" (0,131, que es significativo al nivel de 0,01). En ambos casos, mientras mayor es la presencia positiva de estos factores, mayor es la fuerza con que el profesor experimenta este compromiso. Ahora bien, pese a lo estadísticamente significativo de los coeficientes, hay que advertir también que los valores de la relación son moderados.

\section{Tabla 1}

Matriz de correlaciones entre factores y compromisos

\begin{tabular}{|ll|c|c|c|c|c|}
\cline { 3 - 7 } \multicolumn{1}{c|}{} & \multicolumn{6}{c|}{ Correlaciones } \\
\cline { 2 - 7 } \multicolumn{1}{c|}{} & Logro & $\begin{array}{c}\text { Reconoci- } \\
\text { miento }\end{array}$ & $\begin{array}{c}\text { Recursos } \\
\text { educativos }\end{array}$ & $\begin{array}{c}\text { Condiciones } \\
\text { administrativas }\end{array}$ & $\begin{array}{c}\text { Compromiso } \\
\text { afectivo }\end{array}$ \\
\hline Tau_b de Kendall & Logro & 1,000 &, 002 &, 022 &, $094^{*}$ &, 053 \\
\hline Reconocimiento &, 002 & 1,000 &, 000 &,- 001 &, $127^{* *}$ \\
\hline $\begin{array}{l}\text { Recursos } \\
\text { educativos }\end{array}$ &, 022 &, 000 & 1,000 &,- 016 &, $131^{* *}$ \\
\hline $\begin{array}{l}\text { Condiciones } \\
\text { administrativas }\end{array}$ &, $094^{*}$ &,- 001 &,- 016 & 1,000 &, 077 \\
\hline $\begin{array}{l}\text { Compromiso } \\
\text { afectivo }\end{array}$ &, 053 &, $127^{* *}$ &, $131^{* *}$ &, 077 & 1,000 \\
\hline
\end{tabular}

* La correlación es significativa al nivel 0,05 (bilateral).

** La correlación es significativa al nivel 0,01 (bilateral). 
Por otra parte, es interesante considerar que el factor "Logro" no mantiene relaciones significativas con este compromiso. Es decir, pese a la alta frecuencia que presenta este factor, el COA opera con independencia de él.

Aplicada la regresión logística se obtienen los siguientes resultados:

\section{Tabla 2}

Resumen de los valores de la regresión logística para factores organizacionales y compromiso afectivo

\begin{tabular}{|l|c|c|c|c|c|c|}
\cline { 3 - 6 } \multicolumn{2}{c|}{} & \multicolumn{4}{|c|}{ Porcentajes de aciertos para COA } \\
\hline Factores & Exp-B- & Sig. & Total & $\begin{array}{c}\text { Bajo } \\
\text { compro- } \\
\text { miso }\end{array}$ & $\begin{array}{c}\text { Alto com- } \\
\text { promiso }\end{array}$ & $\begin{array}{c}\mathrm{R}^{2} \text { de Cox } \\
\text { y Snell }\end{array}$ \\
\hline Logro & 0,974 & 0,944 & 53,5 & 0 & 100 & 0,000 \\
Reconocimiento & 1,700 & 0,089 & 56,5 & 57,5 & 55,7 & 0,017 \\
Recursos Educativos & 2,875 & $0,024 * *$ & 62,5 & 69,2 & 56,1 & 0,064 \\
Condiciones Administrativas & 1,188 & 0,153 & 69,2 & 91,4 & 23,5 & 0,039 \\
\hline
\end{tabular}

** sig. menor de 0,05 .

Estos resultados indican que el factor "Recurso Educativo" es el único que se relaciona significativamente con el COA (sig. 0,024). Esta relación implica que los profesores que mencionan tales recursos bajo una orientación positiva tienen un 2,8 veces de ventaja de ubicarse en el grupo de alto compromiso afectivo, que aquellos que lo mencionan con una orientación negativa (Exp. $-\mathrm{B}-=2,875)$. En otras palabras, la orientación que adopte tal factor es significativamente predictora del nivel de compromiso afectivo que presente el profesor.

A partir de estos datos, también se puede observar que el "Recurso Educativo" constituye un factor que predice con más certeza el bajo COA $(69,2 \%)$ que el alto $(56,1 \%)$. Ello significa, que la fuerza del factor en su orientación negativa y la desvinculación afectiva es más potente que la fuerza que se presenta cuando la orientación es positiva.

Por otra parte, es importante señalar que a pesar de ser relevante la relación entre el factor "Recurso Educativo" y el COA, ésta tiene un nivel de ajuste moderado. Ello se expresa en el $\mathrm{R}^{2}$ de Cox y Snell de 0,064, lo cual indica que, a pesar de su significatividad, el factor "Recurso Educativo" constituye una variable muy focalizada para explicar este compromiso. Esto significa que en el comportamiento del COA intervienen otras variables, que no han sido incluidas en este estudio, las cuales pueden aportar mayores cuotas de ajuste para predecir su comportamiento. Este aspecto representa un desafío para futuras investigaciones.

También es interesante considerar que el factor "Reconocimiento" no logra establecer una relación significativa con el COA. Hay que recordar que anteriormente presentó una correlación significativa $(\mathrm{r}=0,127)$. Considerando ambas evidencias, se aplica una prueba de chi-cuadrado para examinar diferencias entre este factor y el COA. 
Los resultados indican que las diferencias no logran ser estadísticamente significativas $\left(\chi^{2}=2,913\right.$ y un $=$ sig. 0,088$)$. De este modo se puede señalar que los resultados de la regresión logística son más confiables y, por consiguiente, habrá que desestimar la relación entre ambas variables.

En el caso de los factores "Logro" y "Condiciones Administrativas" se observa que la orientación que éstos adopten no es estadísticamente significativa para predecir el comportamiento del COA. Ello es corroborado también en la matriz de correlaciones.

En suma, considerando la información obtenida, se puede establecer que el factor que mejor se relaciona con el COA es "Recurso Educativo". La relación que mantiene este factor es corroborada desde la matriz de correlaciones y desde la regresión logística.

\section{CONCLUSION Y DISCUSION}

El presente estudio ha logrado identificar los factores de la organización escolar que son más valorados por los profesores. Ellos son: logro pedagógico, reconocimiento a la labor realizada, recursos educativos y condiciones administrativas. De ellos, el único que presenta una relación significativa con el COA es el "recurso educativo". Antes de hacer referencia a éste, es importante comentar la ausencia de relación con el factor "logro".

Los profesores son altamente sensibles a emocionarse cuando logran -o no logranhacer intervenciones pedagógicas (34\% de los profesores mencionan este factor). Sin embargo, y a pesar de su alta frecuencia, el "logro" no presenta una asociación con el COA.

Es interesante considerar que el "logro" requiere de una actitud proactiva de parte del profesor. El "logro" surge por lo que el profesor hace. Sin embargo, a la luz de los resultados obtenidos, hay que reconocer que este "hacer" no es tan relevante a la hora de evaluar el apego afectivo con el establecimiento.

Esta situación contrasta con los estudios norteamericanos de Kushman (1992) y de Rosenholtz y Simpson (1990). Ellos plantean que el profesor se apega afectivamente al establecimiento debido a las intervenciones psicopedagógicas que desarrolla, lo que a su vez le nutre de un sentimiento de autoeficacia y autoestima profesional. Esta explicación, como otras similares del medio anglosajón, supone un profesor con una actitud eminentemente proactiva: un profesional ávido de experimentar logros pedagógicos, por lo cual siempre está en búsqueda de mayores desafíos educacionales.

A diferencia de los factores que requieren una disposición activa del profesor, como es el "logro" o el "reconocimiento", este estudio ha podido constatar que el COA se configura a partir de las condiciones laborales, donde el docente juega un rol más pasivo. Particularmente el único factor que presenta una relación significativa con el COA es el "recurso educativo".

Es interesante advertir que este factor alude, entre otros aspectos, al perfil académico de los alumnos. Al respecto, el alumno es representado por el docente como una condición laboral que dispone la escuela y que es administrable por la gestión directiva: se puede otorgar, dosificar, sustituir. En cuanto es una condición laboral, los profesores destacan al "buen alumno" como un componente que les reporta tranquilidad para realizar su trabajo y estima profesional. Hay que recordar que los profesores señalan que al recibir 
un "buen recurso educativo", sienten que se los ha respetado y que la administración educacional está preocupada de su dignidad profesional.

Cuando el "recurso educativo" no es lo suficientemente favorable, el profesor desarrolla una significativa desvinculación afectiva. Esta puede ser más potente que la misma vinculación. De acuerdo a los resultados obtenidos, la relación entre "recurso educativo" y COA es más fuerte en un sentido negativo, que positivo.

Siguiendo este análisis, se podría decir que un alumno con dificultades académicas no sólo tiene que sobrellevar su propio fracaso escolar, sino también constituye un referente altamente potente para el descontento emocional del profesor.

Esta situación tiene implicancias extraordinariamente delicadas para los establecimientos que atienden a niños que han experimentado fracasos escolares. Una escuela, en esta situación, podría verse en la dinámica de reducir el descontento emocional evitando los focos de descontento; es decir, el alumno que no es un "buen recurso educativo".

En este sentido, los alumnos que no responden a los rendimientos académicos esperados no representan para el profesor un desafío educativo, sino sólo una condición desfavorable, que hay que intentar evitar. Por consiguiente, las posibilidades que los alumnos progresen, se ven peligrosamente disminuidas en cuanto el profesor sólo lo observa como un obstáculo para su tranquilidad laboral.

Ello plantea entonces un importante desafío para los educadores chilenos: ¿cómo reducir la desvinculación afectiva, sin aniquilar al sujeto de la educación?

\section{BIBLIOGRAFIA}

Allen, N., J. Meyer (1990). The measurement and antecedents of affective, continuance and normative commitment to the organization. Journal of Occupational Psychology, Vol. 63: 1-18.

Becker, H. (1960). Notes on the concept of commitment. American Journal of Psychology, Vol. 66: 32-40.

Castro, E. (2000). La salud del profesor en Chile: antecedentes para un estado del arte. Documento de trabajo del Ministerio de Educación de Chile.

Casen (2000). Información estadística de la población ocupada. En: www.casen.cl

Cornejo, J., E. Rodríguez (1995). Elección y motivos vocacionales, problemas de enseñanza, satisfacción profesional y laboral de profesores de E. Media del Sector municipalizado metropolitano. Proyecto Fondecyt 194-0745.

Etzioni, A. (1961). A comparative analysis of complex organizations. New York: Free Press.

Gatti, B., Y. Esposito, R. Da Silva (1994). Características de los profesores de primer grado en Brasil: Perfil y expectativas. Proyecto Principal de Educación, UNESCO, Boletín 34: 36-44.

Herzberg, F. (1966). The Motivation to Work. Nueva York: John Wiley \& Sons.

Jaros, S. (1997). An assessment of Meyer and Allen's (1991). Three component model of organizational commitment and turnover intentions. Journal of vocational behavior 51: 319-337.

Kushman, J. (1992). The organizational dynamics of teacher workplace commitment: a study of urban elementary and middle school. Educational Administration Quarterly, Vol. 28, 1: 5-42.

Luhmann, N., R. De Georgi (1993). Teoría de la sociedad. Ciudad de México: Universidad Iberoamericana.

Méndez, M., J. Bernales (1996). Factores de estrés en el ejercicio docente de profesores de enseñanza media de establecimientos municipalizados. Boletín de Investigación Educacional, Vol. 11: 204-218.

Mendizábal, N. (1995). Condiciones de trabajo y salud mental de los docentes primarios de la provincia de Buenos Aires. Buenos Aires: Universidad de Buenos Aires. 
Meyer, J., N. Allen (1984). Testing the "side bet theory" of organizational commitment: some methodological considerations. Journal of applied Psychology 69: 372-385.

Meyer, J., N. Allen, C. Smith (1993). Commitment to Organizations and Occupations: extension and test of three component conceptualization. Journal of Applied Psychology, Vol. 78: 538551.

March, J., H. Simon (1977). Teoría de la organización. Barcelona: Ariel.

Rosenholtz, S., C. Simpson (1990). Workplace conditions and the rise and fall of teachers' commitment. Sociology of education, Vol. 63: 241-257.

Rosenblatt, Z. (2001) Teachers multiple roles and skilll flexibility: effects on work attitudes. Journal Educational Administration Quarterly, Vol, 37, 5: 684-708.

Zamora, G. (2005). Permanencia de los profesores en sus establecimientos: análisis desde los compromisos organizacionales y factores de (in)satisfacción. Tesis para obtener el grado de Doctor en Ciencias de la Educación de la Pontificia Universidad Católica de Chile. 
